\title{
Naturally occurring scrapie is associated with a lower CBG binding capacity in ewes
}

\author{
N Picard-Hagen ${ }^{1}$, V Gayrard ${ }^{1}$, M Alvinerie ${ }^{2}$, V Laroute $^{1}$, \\ C Touron ${ }^{1}$, O Andreoletti ${ }^{3}$ and $\mathbf{P}$ L Toutain ${ }^{1}$ \\ ${ }^{1}$ Unité Associée INRA de Pathologie et Toxicologie Expérimentales, Ecole Nationale Vétérinaire de Toulouse, 31076 Toulouse, France \\ ${ }^{2}$ INRA, Station de Pharmacologie, 180 chemin de Tournefeuille 31391 Toulouse, France \\ ${ }^{3}$ Unité Associée INRA de Physiopathologie Infectieuse et Parasitaire des Ruminants, Ecole Nationale Vétérinaire de Toulouse, 31076 Toulouse, France \\ (Requests for offprints should be addressed to P L Toutain, Laboratoire de Physiologie \& Thérapeutique, Ecole Nationale Vétérinaire de Toulouse, \\ 23, chemin des Capelles, 31076 Toulouse, France; Email: pl.toutain@envt.fr)
}

\begin{abstract}
Naturally scrapie-affected ewes present a syndrome of hypercortisolism as evaluated by measuring total plasma cortisol concentrations. The objective of this study was to investigate the plasma protein binding of cortisol and to evaluate the concentration of the biologically active free fraction of cortisol in scrapie-affected ewes. Corticosteroid binding globulin (CBG) binding parameters were evaluated by equilibrium dialysis in 13 naturally scrapie-affected ewes and nine healthy ewes, during two periods of the clinical evolution of the disease. The hypercortisolism of the scrapie-affected ewes was confirmed by a significant increase of the plasma $20 \beta$-dihydrocortisol and cortisone concentrations, while total cortisol concentrations, obtained from an isolated sample, did not differ between scrapie-affected and control ewes. The scrapie diagnosis was confirmed by histopathology. The CBG maximal capacity $\left(B_{\max }\right)$ was two times lower in scrapieaffected ewes than in healthy ewes $(37 \pm 32 \mathrm{nM}$ and
\end{abstract}

$73 \pm 28 \mathrm{nM}$ respectively). The dissociation constant $K_{\mathrm{d}}$ $(8 \cdot 8 \pm 3.7 \mathrm{nM}$ and $9 \cdot 8 \pm 3.0 \mathrm{nM}$ respectively) and the non-specific constant value of binding to albumin $(1 \cdot 13 \pm 0.18$ and $1 \cdot 14 \pm 0.23$ respectively) did not differ significantly between diseased and control ewes. The significant increased concentrations of CBG-free cortisol (i.e. both albumin-bound and free cortisol fractions) in scrapie-affected ewes indicates that total plasma cortisol concentration is not an appropriate index of pituitary-adrenocortical hyperactivity.

In conclusion, ewes with naturally occurring scrapie display a syndrome of hypercortisolism associated with a lower CBG binding capacity which leads to an overexposure of glucocorticoid-sensitive targets to CBG-free cortisol. The physiopathological consequences of this overexposure on the development of the neurodegenerative process in prion disease are discussed.

Journal of Endocrinology (2000) 165, 527-532

\section{Introduction}

Scrapie is an ovine subacute transmissible spongiform encephalopathy (TSE) which belongs to a group of slowly progressive neurological disorders including CreutzfeldtJakob disease and bovine spongiform encephalopathy. These TSE are caused by an unconventional transmissible agent and characterized by the accumulation of PrPsc, an altered isoform of a normal cellular protein designated prion protein $(\mathrm{PrP})$. In a previous study, we demonstrated that plasma and urinary concentrations of corticoids were much higher in natural scrapie-affected ewes than in healthy ewes (Schelcher et al. 1999), this hypercortisolism being associated with hypertrophy of the adrenal gland. In sheep, more than $80 \%$ of circulating cortisol is bound to the corticosteroid binding globulin (CBG or transcortin) (Gayrard et al. 1996), a glycoprotein that binds cortisol with high affinity but low capacity, and to serum albumin with a much lower affinity (Siiteri et al. 1982). It is generally accepted that the biological activity of cortisol is determined by the free or albumin-bound fraction rather than by the CBG-bound fraction in the circulation (Rosner 1990, 1991). In practice, the measurement of total cortisol concentrations is generally used to evaluate the activity of the hypothalamo-pituitary-adrenal axis, although it only indirectly reflects the adrenal activity. Different reports suggest that plasma CBG concentrations or binding capacity are not constant and can be altered by many factors such as stress, inflammation, estrogens or glucocorticoid treatment (Westphal 1971, Smith \& Hammond 1992). Moreover, it has been shown that minor alterations in the concentrations of CBG may cause major changes in free cortisol (Dunn et al. 1981). For instance, in certain situations like stress, changes in free cortisol levels were evidenced without any modification in the basal concentration of total cortisol (Alexander \& Irvine 1998). 
In order to assess the extent of the adrenal hyperactivity in scrapie-affected ewes, the potential availability of free cortisol to target tissues had to be evaluated by measuring the binding properties of CBG. The purpose of this experiment was to compare the cortisol binding parameters in scrapie-affected ewes with those of control healthy animals and to examine the time development of these parameters during the clinical phase of scrapie.

\section{Materials and Methods}

\section{Animals}

Nine control and 13 naturally scrapie-affected red head Manech ewes were investigated. The ages of both healthy and diseased ewes ranged from 1 to 4 years. The diagnosis of scrapie, based on classical clinical signs, i.e. pruritus, behavioral changes, tremor and locomotor incoordination, was established before the beginning of the experiments. The healthy ewes were included in the trial on the basis of an absence of any clinical signs of scrapie. None of the classical clinical signs of scrapie has since been observed in these ewes during the 6 months following the sampling periods. The scrapie-affected ewes were sacrificed when they manifested the final clinical stages of scrapie, i.e. when the clinical signs had progressed to irreversible recumbency.

\section{Design}

Two blood samples were collected at about 1 month interval during the clinical phase of scrapie in order to determine CBG binding parameters. Another blood sample, collected about 1 month before or after these sampling sessions, was used to evaluate plasma cortisol metabolites levels in order to confirm the hypercortisolism syndrome in scrapie-affected ewes and to calculate the different cortisol fractions from individual analysis of CBG parameters.

\section{Blood sampling}

Thirty or $50 \mathrm{ml}$ blood samples were obtained by venipuncture of the left jugular vein in order to measure in vitro the plasma protein binding of cortisol and plasma corticoids concentrations. Blood was collected in heparinized tubes and centrifuged at $1200 \boldsymbol{g}$ for $10 \mathrm{~min}$. Plasma was stored at $-20{ }^{\circ} \mathrm{C}$ until assayed.

\section{Analytical methods}

Plasma corticoid (cortisol, 20 $\beta$-dihydrocortisol, cortisone) concentrations were determined using an adapted HPLC after methylene chloride extraction as previously described (Alvinerie \& Toutain 1982). Within- and between-day precisions were lower than 15\%. The accuracy range was $89-105 \%$ and the extraction recovery ranged between 75 and $85 \%$. The validated quantification limit of the assay was $1.25 \mathrm{ng} / \mathrm{ml}$ for all analytes.

\section{Histology}

The diagnosis of scrapie was confirmed by the identification of perikaryonic and/or neuropilar vacuolization in at least three grey matter nuclei as described previously (Schelcher et al. 1999).

\section{Binding experiments}

The endogenous corticoids were removed from plasma by adsorption on charcoal (Alvinerie et al. 1988). The plasma samples, free of steroid, were chilled at $-4{ }^{\circ} \mathrm{C}$ until the in vitro cortisol binding study was carried out either the same or next day. In vitro protein binding of cortisol was measured over a wide range of concentrations $(0.0055$ to $2.762 \mu \mathrm{M}$ ) by equilibrium dialysis using a Dianorm system (CH8135, Langenau, Zurich, Switzerland) as previously described by Gayrard et al. (1996).

\section{Data analysis}

Protein-bound cortisol concentrations were plotted against the unbound concentrations. For all ewes, the profiles indicated the presence of both saturable and non-saturable protein binding, i.e. transcortin and albumin respectively. These data were fitted by use of the following equation,

$$
\mathrm{B}=\frac{\mathrm{B}_{\max } \times \mathrm{F}}{\mathrm{Kd}+\mathrm{F}}+\mathrm{NS} \times \mathrm{F}
$$

where $B$ and $F$ are the molar concentrations of bound and free cortisol respectively. $B_{\max }(\mathrm{M})$ and $K_{\mathrm{d}}(\mathrm{M})$ are the CBG maximal capacity and the dissociation constant of binding respectively. $B_{\max }$ is equivalent to the product of the number of binding sites of the specific binding protein $(\mathrm{CBG})$ and its molar concentration, i.e. the concentration of the CBG binding sites. NS is a dimensionless non-specific binding constant of cortisol related to albumin.

Binding parameters $\left(B_{\max }, K_{\mathrm{d}}\right.$ and NS) were evaluated by a computerized nonlinear least squares regression program adapted from Multi (Yamaoka et al. 1981).

The concentrations of free, albumin-bound and CBGbound cortisol fractions for measured plasma cortisol and different concentrations of total cortisol $(2,10,20,50$ and $100 \mathrm{ng} / \mathrm{ml}$ ) were evaluated as described previously by Gayrard et al. (1996).

\section{Statistical analysis}

Results are reported as mean \pm S.D. or as medians and range. Statistics were performed using the Statgraphics 
Table 1 Corticoid plasma levels in eight healthy and 13 naturally scrapie-affected ewes.

Values are medians and ranges. Plasma corticoid concentrations were determined using an HPLC technique

\section{Plasma corticoid concentrations}

Cortisol (nM)

20ß-dihydrocortisol (nM)

Cortisone (nM)

\begin{tabular}{l} 
Healthy ewes \\
\hline $51(26-236)$ \\
$4 \cdot 8(1 \cdot 6-8 \cdot 3)$ \\
$8 \cdot 3(5 \cdot 2-19 \cdot 9)$
\end{tabular}

Scrapie-affected ewes

$57(24-360)$

$8 \cdot 1(3 \cdot 2-48 \cdot 3)^{* *}$

$25(1 \cdot 7-71 \cdot 1)^{*}$

The effect of scrapie on corticoid concentrations was determined by a nonparametric Kruskal-Wallis test. ${ }^{*} P<0 \cdot 05,{ }^{*} P<00 \cdot 02$.

program (version 5, STSC Inc. 1991). A P value lower than 0.05 was considered as significant. Plasma corticoid concentrations below the limit of quantification of the HPLC were arbitrarily fixed at $0.6 \mathrm{ng} / \mathrm{ml}$. The influence of scrapie on corticoid plasma concentrations was assessed using the unpaired Student's $t$-test. The nonparametric Kruskal-Wallis test was used to analyze the effect of scrapie on corticoid concentrations, due to the variability of the parameters between animals and the relatively small number of subjects.

The CBG binding parameters were analyzed using an ANOVA with three factors: group (healthy or scrapie-affected ewes), ewe within group and period.

The levels of different cortisol fractions (i.e. free, bound to $\mathrm{CBG}$ or bound to albumin) calculated from individual CBG and albumin binding parameters were analyzed by analysis of covariance (ANCOVA) with measured cortisol as covariate and the factor group (healthy or scrapie-affected ewes).

\section{Results}

Corticoid plasma concentrations in scrapie-affected and in control ewes

No evidence of behavioral suffering was recorded during the two sampling periods and scrapie-affected ewes behaved and ate normally since they were excluded from the experiment as soon as severe clinical signs developed.

Table 1 shows the medians and ranges of plasma corticoid concentrations of control and scrapie-affected ewes. The median of plasma $20 \beta$-dihydrocortisol concentrations was about two times higher in scrapie-affected ewes $(8 \cdot 1 \mathrm{nM}, 3 \cdot 2-48 \mathrm{nM})$ than that obtained in healthy ewes $(4 \cdot 8 \mathrm{nM}, 1 \cdot 6-8 \cdot 3 \mathrm{nM})$. A Kruskal-Wallis analysis showed a significant effect of scrapie on plasma $20 \beta-$ dihydrocortisol and cortisone concentrations $(P<0.02$ and $P<0.05$ respectively), while the median plasma cortisol of scrapie-affected ewes ( $57 \mathrm{nM}, 24-360 \mathrm{nM}$ ) did not differ significantly from values observed in healthy ewes $(51 \mathrm{nM}$, 26-236 nM).
Protein-binding parameters of cortisol in scrapie-affected and in control ewes

The mean ( \pm s.D.) protein-binding parameters of cortisol during the two blood sampling periods are presented in Table 2. The ANOVA showed a significant effect of scrapie on $B_{\max }$ (ANOVA, $P<0 \cdot 01$ ) but not for $K_{\mathrm{d}}$ and NS $(P>0 \cdot 05)$. The analysis of variance revealed a statistically significant effect of blood sampling period on $K_{\mathrm{d}}(P<0 \cdot 05)$ and NS $(P<0 \cdot 005)$, but the difference was not relevant in a physiologic context. There was no similar period effect for $B_{\max }(P=0 \cdot 19$, ANOVA $)$. The overall mean maximal binding capacity of transcortin in scrapie-affected ewes $(37 \pm 32 \mathrm{nM})$ was about two times lower than that obtained in healthy ones $(73 \pm 29 \mathrm{nM})$. The overall mean CBG $K_{\mathrm{d}}$ was $8.8 \pm 3.7 \mathrm{nM}$ in scrapie-affected ewes and $9 \cdot 8 \pm 3 \cdot 0 \mathrm{nM}$ in healthy ones. The overall mean NS was $1 \cdot 13 \pm 0.18$ in scrapie-affected ewes and $1 \cdot 14 \pm 0.23$ in healthy ones.

Concentrations of unbound and protein-bound cortisol in scrapie-affected and in control ewes

Free cortisol, CBG-bound cortisol and albumin-bound cortisol were calculated from individual analysis of $\mathrm{CBG}$ binding parameters obtained in period 1 and actual measured plasma cortisol levels. Free cortisol concentrations observed in the present experiment for scrapieaffected ewes ranged between 4.6 and $150 \mathrm{nM}$ (median: $23 \mathrm{nM}$ ) and tended to be higher than those of healthy ewes which ranged between $2 \cdot 4$ and $68 \mathrm{nM}$ (median: $8.8 \mathrm{nM})$, but the difference did not reach significance (Kruskal-Wallis, $P=0 \cdot 15$ ). However, the ANCOVA showed that free cortisol levels, for a given measured total cortisol concentration, were significantly higher in scrapieaffected than in healthy sheep $(P<0 \cdot 01)$. Similarly, the albumin-bound cortisol concentrations of scrapie-affected ewes ranged between 4.3 and $207 \mathrm{nM}$ (median: $27 \mathrm{nM}$ ) and tended to be higher than those of healthy ewes which ranged between $2 \cdot 8$ and $92 \mathrm{nM}$ (median: $11 \mathrm{nM}$ ), but the difference did not reach significance (KruskalWallis, $P=0 \cdot 19)$. However, for albumin-bound cortisol 
Table 2 Plasma protein-binding parameters in nine healthy and 13 naturally scrapie-affected ewes in the first blood sampling period and in six healthy and six diseased ewes in the second blood sampling period. $B_{\max }$ and $K_{\mathrm{d}}$ are the CBG maximal capacity and its dissociation constant respectively. $B_{\max }$ is equivalent to the product of the number of binding sites by the molar concentration of the protein, i.e. the concentration of the CBG binding site concentration. NS is a dimensionless proportionality constant of the nonspecific binding of cortisol. Values are means \pm S.D. For both $B_{\max }$ there was no period effect and scrapie had a significant effect $(P<0 \cdot 01)$ for both periods. For $K_{d}$ and NS, there was a significant period effect $(P<0.05$ and $P<0.005$ respectively), but scrapie had no significant effect $(P>0.05)$

\begin{tabular}{|c|c|c|c|c|}
\hline \multirow[b]{2}{*}{$\begin{array}{l}\text { Protein-binding } \\
\text { parameters }\end{array}$} & \multicolumn{2}{|c|}{ Healthy ewes } & \multicolumn{2}{|c|}{ Scrapie-affected ewes } \\
\hline & $\begin{array}{l}\text { Period } 1 \\
(n=9)\end{array}$ & $\begin{array}{l}\text { Period } 2 \\
(n=6)\end{array}$ & $\begin{array}{l}\text { Period } 1 \\
(n=13)\end{array}$ & $\begin{array}{l}\text { Period } 2 \\
(n=6)\end{array}$ \\
\hline $\begin{array}{l}B_{\max (\mathrm{nM})} \\
K_{\mathrm{d}}(\mathrm{nM}) \\
\mathrm{NS}\end{array}$ & $\begin{array}{c}66 \pm 25 \\
8 \cdot 5 \pm 1 \cdot 2 \\
1 \cdot 24 \pm 0 \cdot 15\end{array}$ & $\begin{aligned} 82 & \pm 30 \\
11 \cdot 8 & \pm 3 \cdot 9 \\
0 \cdot 99 & \pm 0 \cdot 25\end{aligned}$ & $\begin{array}{c}31 \pm 34 \\
8 \cdot 0 \pm 4 \cdot 0 \\
1 \cdot 19 \pm 0 \cdot 16\end{array}$ & $\begin{array}{c}48 \pm 24 \\
10 \cdot 5 \pm 2 \cdot 2 \\
1 \cdot 01 \pm 0 \cdot 19\end{array}$ \\
\hline
\end{tabular}

concentrations, ANCOVA showed that the effect of scrapie, for a given measured total cortisol concentration, was very close to significance $(P=0 \cdot 07)$. The CBG-bound cortisol concentrations of scrapie-affected ewes ranged between $2 \cdot 0$ and $80 \mathrm{nM}$ (median: $12 \mathrm{nM}$ ) and were significantly lower than those of healthy ewes which ranged between 16 and $75 \mathrm{nM}$ (median: $29 \mathrm{nM}$, KruskalWallis, $P<0 \cdot 02)$. When the influence of the covariate, total measured cortisol concentrations, was removed by ANCOVA, the difference between scrapie-affected sheep and healthy sheep was very close to significance (ANCOVA, $P=0 \cdot 06$ ).

Figure 1 shows the predicted plasma concentrations (mean \pm s.D.) of free cortisol, albumin-bound and CBGbound cortisol fractions for different total plasma cortisol levels ranging from 5.5 to $276 \mathrm{nM}$, in healthy and scrapie-affected ewes. The mean free cortisol levels for mean plasma cortisol concentrations observed in healthy ewes, i.e. from 14 to $28 \mathrm{nM}$ (Schelcher et al. 1999), varied from 1.5 to $3.9 \mathrm{nM}$, and for mean plasma cortisol concentrations ranging between 28 and $165 \mathrm{nM}$ in scrapieaffected ewes (Schelcher et al. 1999), the mean free cortisol levels varied from $7 \cdot 9$ to $64 \mathrm{nM}$.

\section{Discussion}

The main result of the present study is that the increase in plasma corticoid concentrations in scrapie-affected ewes is also associated with a significant decrease, by a factor of two, of the binding of cortisol to the transcortin, and an increase of the CBG-free cortisol (i.e. both albuminbound and free cortisol fractions) concentration which is more than proportional to the observed increase of total plasma cortisol concentrations.

In our experiment, most of the plasma sample levels of cortisol in healthy ewes were elevated compared with the range of physiological concentrations (Schelcher et al.
1999), this elevation could result from the stress associated with the sampling procedure (Fulkerson \& Jamieson 1982). Plasma concentrations of total cortisol did not differ between scrapie-affected and healthy ewes in this experiment, whilst plasma $20 \beta$-dihydrocortisol and cortisone levels were significantly increased in naturally scrapieaffected ewes. Indeed, the plasma cortisol concentrations are subject to fluctuations of endogenous (pulsatility) or exogenous (e.g. in response to a stimulus such as the acute stress of the sampling procedure) origin. Thus, it is difficult with an isolated sample to show a difference between pathological status. These fluctuations did not exist for cortisol plasma or urine metabolites due to the dampening effect of the metabolic process on the short-term variations of cortisol secretion (Schelcher et al. 1999). When the plasma cortisol metabolites (20 $\beta$-dihydrocortisol, cortisone) are taken into account, the results of the present experiment are consistent with the observed stimulation of the adrenal function in scrapie-affected ewes demonstrated in a previous study (Schelcher et al. 1999) and the enlargement of the adrenal cortex observed in scrapieaffected ewes (Beck et al. 1964), in experimentally infected mice (Kim et al. 1988) and in hamsters infected with the 139H scrapie strain (Carp et al. 1990).

The CBG binding parameters of healthy ewes varied within the range of physiological values previously obtained by the same standardized equilibrium dialysis method (Gayrard et al. 1996). We have shown that the maximal binding capacity of transcortin in scrapie-affected ewes was decreased by a factor of two whereas protein binding parameters, namely $K_{\mathrm{d}}$ and NS, were not modified, suggesting that the affinity properties of CBG were unchanged in the prion disease.

The lower CBG binding capacity of scrapie-affected ewes could be related to the negative effect of hypercorticism on CBG synthesis as previously suggested in rats or in humans receiving glucocorticoids or in Cushing's disease (Schlechte \& Hamilton 1987, Fleshner et al. 1995). 

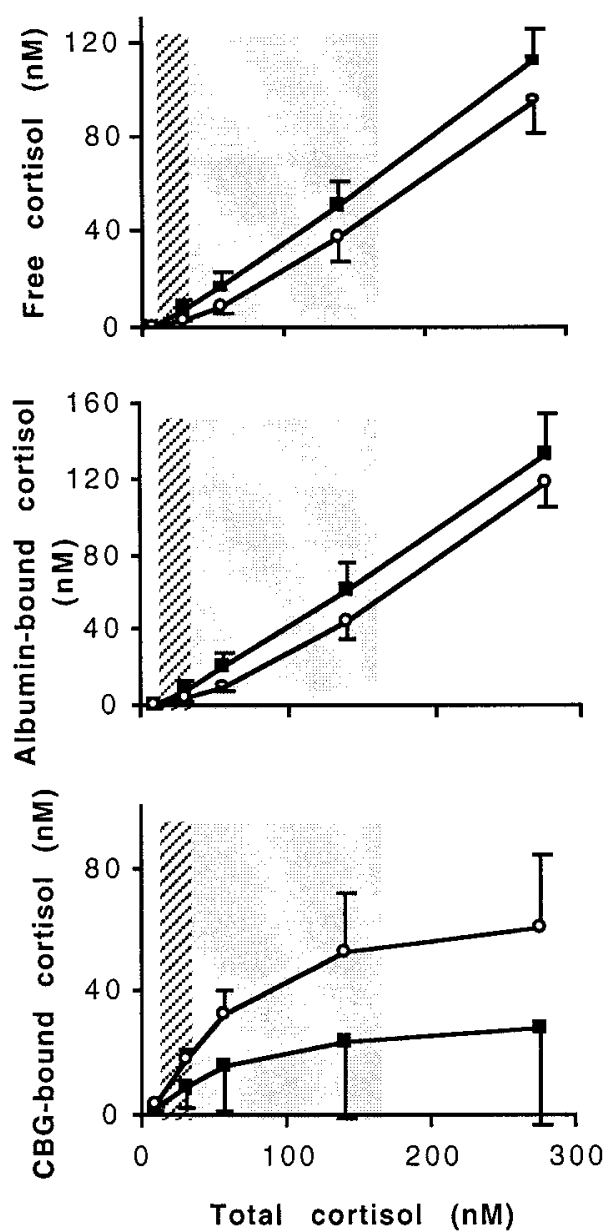

Figure 1 Concentrations (means \pm S.D.) of free, albumin-bound and CBG-bound cortisol in healthy $(\bigcirc, n=9)$ and scrapie-affected $(\boldsymbol{\square}, n=13)$ ewes, calculated for five standard total cortisol concentrations ranging from 5.5 to $276 \mathrm{nM}$. Concentrations were calculated from the individual estimated plasma protein-binding parameters obtained in period 1 . The hatched area represents range of mean cortisol levels observed in healthy ewes $(12-28 \mathrm{nM})$ and the shaded area represents range of mean plasma cortisol levels in scrapie-affected ewes (28-165 nM) (Schelcher et al. 1999).

Indeed, the sustained elevation of serum levels of glucocorticoids might lead to a decrease in protein synthesis, as corticosteroids in particular are known to suppress hepatic CBG production in rats (Feldman et al. 1979, Smith \& Hammond 1992). We cannot exclude the hypothesis that another scrapie-induced dysfunction could result in lower CBG maximal binding capacity.

The relative distribution of cortisol between the protein-bound and the free fractions depends on both plasma protein binding parameters and the actual rate of cortisol secretion. The free plasma concentration of cortisol depends only on two physiological processes, namely the rate of adrenal cortisol secretion and the plasma clearance of free cortisol. It was shown that plasma free cortisol clearance was unchanged in scrapie-affected ewes and that the increase of free cortisol concentrations was only due to an overproduction of cortisol by the adrenal gland (Gayrard et al. 2000). In other words, the actual free cortisol concentration is not controlled by CBG or albumin binding parameters. In contrast, the total plasma cortisol concentration, which is actually measured and interpreted, is controlled by the binding properties of free cortisol to CBG and albumin and this relationship has to be taken into account when interpreting a measured plasma concentration, which is a total plasma cortisol concentration.

The $B_{\max }$ of transcortin was two times lower in scrapieaffected ewes than in healthy ewes; the physiological meaning of this decrease remains unclear and it must be realized that the interpretation of a given plasma cortisol concentration for healthy and scrapie-affected ewes will be different, e.g. for a plasma cortisol concentration of $28 \mathrm{nM}$ it can be ascertained that the free cortisol concentration is about $3.9 \mathrm{nM}$ in healthy ewes whereas it is $7.9 \mathrm{nM}$ (about two times higher) in scrapie-affected ewes.

The relative distribution of cortisol between the protein-bound and the free fractions was modified in scrapie-affected ewes. The CBG-bound cortisol was significantly lower in scrapie-affected than in healthy ewes; the physiological meaning of this decrease remains unclear, but several reports suggest that cortisol-bound CBG could have a, not yet elucidated, biological function other than the simple transport of cortisol (Hryb et al. 1986, Rosner 1991).

The meaning and consequences of the increase of the free cortisol fraction in naturally scrapie-affected ewes should be taken into consideration. It is generally thought that only the CBG-free fraction of cortisol can cross the blood-brain barrier (Rosner 1990, Hammond 1990) and that CBG-free serum cortisol is of the same order of magnitude as the cerebrospinal fluid (CSF) cortisol levels (Martensz et al. 1983, Schwarz \& Pohl 1992). In addition, previous studies in monkeys showed a disproportionate elevation in CSF cortisol when blood levels of the hormone were persistently elevated by repeated injections of either cortisol or adrenocorticotropin (ACTH), due probably to an accumulation of cortisol within CSF (Martensz et al. 1983). These observations raise the question of the effect, in scrapie-affected sheep, of a putative chronic overexposure to a high level of free cortisol in the central nervous system, prolonged elevated corticosteroid exposure leading to neurodegenerative loss of neurons in the rodent brain regions possessing high concentrations of glucocorticoid receptors and particularly in the hippocampus (Sapolsky et al. 1985). It can therefore be hypothesized that in the prion diseases, saturated CBG associated with a long-term sustained hypersecretion of glucocorticoids could exacerbate the neurodegenerative process. 


\section{Acknowledgements}

The authors thank J F Sutra and N Gauthier for technical assistance.

\section{References}

Alexander SL \& Irvine CHG 1998 The effect of social stress on adrenal axis activity in horses: the importance of monitoring corticosteroidbinding globulin capacity. Journal of Endocrinology 157 425-432.

Alvinerie M \& Toutain PL 1982 Simultaneous determination of corticosterone, hydrocortisone, dexamethasone in dog plasma using high performance liquid chromatography. Journal of Pharmaceutical Sciences 71 816-818.

Alvinerie M, Houin G \& Toutain PL 1988 Prednisolone binding to plasma proteins in domestic species. Journal of Pharmaceutical Sciences 77 937-938.

Beck E, Daniel PM \& Parry HB 1964 Degeneration of the cerebellar and hypothalamo-neurohypophysial systems in sheep with scrapie and its relationship to human system degenerations. Brain $\mathbf{8 7}$ 153-176.

Carp RI, Kim YS \& Callahan SM 1990 Pancreatic lesions and hypoglycemia-hyperinsulinemia in scrapie-injected hamsters. Journal of Infectious Diseases 161 462-466.

Dunn JF, Nisula BC \& Rodbard D 1981 Transport of steroid hormones: binding of 21 endogenous steroids to both testosteronebinding globulin and corticosteroid-binding globulin in human plasma. Journal of Clinical Endocrinology and Metabolism 53 58-68.

Feldman D, Mondon CE, Horner JA \& Weiser JN 1979 Glucocorticoid and estrogen regulation of corticosteroid-binding globulin production by rat liver. American Journal of Physiology 237 E493-E499.

Fleshner M, Deak T, Spencer RL, Laudenslager ML, Watkins LR \& Maier SF 1995 A long term increase in basal levels of corticosterone and a decrease in corticosteroid-binding globulin after acute stressor exposure. Endocrinology 136 5336-5342.

Fulkerson WJ \& Jamieson PA 1982 Pattern of cortisol release in sheep following administration of synthetic ACTH or imposition of various stressor agents. Australian Journal of Biological Sciences 35 $215-222$.

Gayrard V, Alvinerie M \& Toutain PL 1996 Interspecies variations of corticosteroid-binding globulin parameters. Domestic Animal Endocrinology 13 35-45.

Gayrard V, Picard-Hagen N, Grino M, Sauze N, Grandjean C, Galea J, Andredetti O, Schelcher F \& Toutain PL 2000 Major hypercorticism is an endocrine feature of ewes with naturally occurring scrapie. Endocrinology 141 (In Press).
Hammond GL 1990 Molecular properties of corticosteroid-binding globulin and sex-steroid binding proteins. Endocrine Reviews $\mathbf{1 1}$ 65-79.

Hryb DJ, Kahn MS, Romas NA \& Rosner W 1986 Specific binding of human corticosteroid-binding globulin to cell membranes. Proceedings of the National Academy of Sciences of the USA 83 3253-3256.

Kim YS, Carp RI, Callahan SM \& Wisniewski HM 1988 Adrenal involvement in scrapie-induced obesity. Proceedings of the Society for Experimental Biology and Medicine 189 21-27.

Martensz ND, Herbert J \& Stacey PM 1983 Factors regulating levels of cortisol in cerebrospinal fluid of monkeys during acute and chronic hypercortisolemia. Neuroendocrinology 36 39-48.

Rosner W 1990 The functions of corticosteroid-binding globulin and sex hormone-binding globulin: recent advances. Endocrine Reviews 11 80-91.

Rosner W 1991 Plasma steroid-binding proteins. Endocrinology and Metabolism Clinics of North America 20 697-720.

Sapolky RM, Krey LC \& McEwen BS 1985 Prolonged glucocorticoid exposure reduces hippocampal neuron number: implications for aging. Journal of Neuroscience $\mathbf{5}$ 1221-1227.

Schelcher F, Picard-Hagen N, Laroute V, Gayrard V, Popot MA, Andreoletti O \& Toutain PL 1999 Corticoid concentrations are increased in the plasma and urine of naturally occurring scrapie in ewe. Endocrinology 140 2422-2425.

Schlechte JA \& Hamilton D 1987 The effect of glucocorticoids on corticosteroid binding globulin. Clinical Endocrinology 27 197-203.

Schwarz S \& Pohl P 1992 Steroid hormones and steroid hormone binding globulins in cerebrospinal fluid studied in individuals with intact and with disturbed blood-cerebrospinal fluid barrier. Neuroendocrinology 55 174-182.

Siiteri PK, Murai JT, Hammond GL, Nisker JA, Raymoure WJ \& Kuhn RW 1982 The serum transport of steroid hormones. Recent Progress in Hormone Research 38 457-510.

Smith CL \& Hammond GL 1992 Hormonal regulation of corticosteroid-binding globulin biosynthesis in the male rat. Endocrinology 130 2245-2251.

Westphal U 1971 Corticosteroid-binding globulin (CBG) of man and other species. In Steroid-Protein Interactions, pp 164-355. Eds F Gross, A Labhart, T Mann, LT Samuels \& J Zander. New York: Springer-Verlag.

Yamaoka K, Tanigawara K, Nakagawa T \& Uno T 1981 A pharmacokinetic analysis program (multi) for microcomputer. Journal of Pharmacobiodynamics 4 879-889.

Received 2 August 1999

Accepted 2 December 1999 